\title{
Principios de diseño de tareas en Socioepistemología
}

\author{
Task design principles in Socioepistemology \\ Jesús Eduardo Hinojos Ramos \\ Fabián Wilfrido Romero Fonseca \\ Rosa María Farfán Márquez
}

\begin{abstract}
RESUMEN
Proponemos una postura acerca de los principios de diseño de tareas en Matemática Educativa desde la Socioepistemología, tomando como premisa la reflexión que diversos grupos de investigación han hecho acerca de la forma de fundamentar los diseños de intervención en el aula y para la investigación. Realizamos una vinculación entre los principios de diseño en marcos de nivel intermedio y de dominio específico con los principios de la Teoría Socioepistemológica y la problematización del saber matemático. Además mostramos dos ejemplos de investigaciones dentro de este marco teórico, uno enfocado en la serie trigonométrica de Fourier en su contexto de origen y otro enmarcado en la ingeniería eléctrica como práctica de referencia para la resignificación del estado estacionario, donde se ilustra cómo los principios de diseño de tareas permiten fundamentar las situaciones de aprendizaje de ambos proyectos de investigación. Concluimos que la problematización del saber matemático juega un papel importante en la construcción de los principios de diseño, ya que con esta se proponen rediseños del discurso matemático escolar.
\end{abstract}

Palabras clave: matemática educativa, diseño instruccional, análisis epistemológico, análisis histórico, método cualitativo.

\begin{abstract}
A position about the principles of task design in Educational Mathematics from Socio-epistemology is proposed, taking as a premise the reflection done from diverse research groups regarding how task-designs for research and school intervention are justified and carried out. We make an articulation between the design principles in intermediate level and domain-specific frames with the principles of the Socioepistemological Theory and the problematization of the mathematical knowledge. In addition, two examples of research within this theoretical framework are shown, one focused on the Fourier series in its context of origin and another one in electrical engineering as a referral practice for the resignification of the steady-state, both examples help to illustrate how task-design principles give foundation to the learning situations of both research projects. We conclude that the problematization of the mathematical knowledge plays an important role on the construction of design principles, as the problematization proposes re-designs for the school mathematics discourse.

Keywords: mathematics education, instructional design, epistemological analysis, historical analysis, qualitative method.
\end{abstract}




\section{INTRODUCCIÓN}

En la actualidad, diversos grupos de investigación y comisiones internacionales en matemática educativa se han preocupado por reflexionar acerca de la forma en la que se realiza el diseño de tareas, tanto para investigación como para el aula de matemáticas - por ejemplo, el ICMI study 22 (Whatson y Ohtani, 2015) y los seminarios de diseño de tareas de la Sociedad Matemática Española-, bajo premisas de ¿por qué se realiza un diseño?, ¿qué métodos y principios guían la construcción de un diseño?, ¿qué se puede conocer de un grupo social a partir de las tareas en investigación?; desde distintos posicionamientos teóricos, cada uno con sus propias metodologías y principios, se busca responder estas preguntas (García, 2019).

Respecto de los posicionamientos teóricos que sustentan los diseños, Kieran, Doorman y Ohtani (2015) los clasifican en términos de su alcance o nivel de detalle en: 1) grandes marcos - grandes teorías que se deben adaptar e interpretar para cubrir las necesidades de la investigación de diseño, por ejemplo, el socioconstructivismo como teoría de aprendizaje-, 2) marcos de nivel intermedio -si bien forman parte de los grandes marcos, son más especializados que estos y se pueden aplicar a una gran variedad de áreas de las matemáticas, por ejemplo, la Matemática Educativa y la Teoría Socioepistemológica dentro de ella, con sus principios filosóficos que la fundamentan-, 3) marcos de dominio específico -a diferencia de los marcos de nivel intermedio, estos abordan conceptos, procedimientos o procesos particulares, matemáticos en el caso de la matemática educativa- y 4) marcos relacionados con características particulares del entorno de aprendizaje -por ejemplo los marcos para el uso de herramientas/mediadores-.

Jesús Eduardo Hinojos Ramos. Centro de Investigación y de Estudios Avanzados del Instituto Politécnico Nacional, México. Es Maestro en Matemática Educativa e Ingeniero en Electrónica por el Instituto Tecnológico de Sonora. Entre sus publicaciones más recientes se encuentran los artículos en coautoría "La analogía entre el calor y la electricidad. Una base para confrontar el obstáculo epistemológico sustancialista en la electricidad en escuelas de ingeniería" (2018) y "Acerca de las nociones de estabilidad en electricidad, la relación entre el calor y la electricidad” (2017). Su línea de investigación es Construcción social del pensamiento matemático avanzado. Correo electrónico: jesus.hinojos@cinvestav.mx. ID: https://orcid.org/0000-0003-3276-0322.

Fabián Wilfrido Romero Fonseca. Universidad de Costa Rica. Es Maestro en Ciencias con especialidad en Matemática Educativa por el Cinvestav-IPN y Licenciado en Enseñanza de las Matemáticas por la Universidad de Costa Rica. Entre sus publicaciones recientes se encuentra el artículo en coautoría "Learning situation for the trigonometric Fourier series from a Socio-epistemological stand point” (2019). Su línea de investigación es Construcción social del pensamiento matemático avanzado. Correo electrónico: fabian.romero@ucr.ac.cr. ID: https://orcid.org/0000-0003-4472-963X.

Rosa María Farfán Márquez. Investigadora titular del Departamento de Matemática Educativa del Centro de Investigación y de Estudios Avanzados del Instituto Politécnico Nacional, México. Es Doctora y Maestra en Ciencias con Especialidad en Matemática Educativa por el Cinvestav-IPN. Pertenece al Sistema Nacional de Investigadores, Nivel II. Entre sus publicaciones recientes se encuentra el artículo en coautoría "El desarrollo del talento de las mujeres en matemáticas desde la Socioepistemología y la perspectiva de género: un estudio de biografías” (2018). Correo electrónico: rfarfan@cinvestav.mx. ID: https:// orcid.org/0000-0003-1229-8521. 
El objetivo de este escrito es dar evidencia respecto de los principios de diseño de tareas para marcos de nivel intermedio y de dominio específico. Concretamente se pretende evidenciar la relación entre los fundamentos de la Socioepistemología y la problematización del saber matemático con los principios de diseño de tareas en los dominios intermedio y específico, a través de ejemplos de investigación relativos al estado estacionario.

\section{LA Socioepistemología Y LOS PRINCIPIOS DE DISEÑO DE TAREAS EN MARCOS DE DOMINIO INTERMEDIO}

La Teoría Socioepistemológica de la Matemática Educativa o Socioepistemología (Cantoral, 2013) es un marco teórico que estudia la construcción social del conocimiento matemático y su difusión institucional. La Socioepistemología considera a las prácticas sociales como las generadoras del conocimiento matemático, y por lo tanto que el conocimiento matemático es un emergente de las dinámicas sociales, por lo que las prácticas situadas anteceden y acompañan la construcción del conocimiento matemático.

Por otra parte, como fruto del proceso de transposición didáctica, los objetos matemáticos sufren una serie de transformaciones hasta tornarse objetos de enseñanza (Chevallard, 1997). Ante este hecho, la Socioepistemología sostiene que cuando el conocimiento llega a la escuela se producen diferentes discursos - constructo teórico de discurso matemático escolar (dME) - que cambian la organización y funcionamiento del conocimiento matemático (Cantoral, 2013).

El dME es un sistema de razón que excluye tanto a estudiantes como profesores de la construcción social de conocimiento matemático, lo cual se evidencia a través de las siguientes características: el carácter utilitario y no funcional del conocimiento, la atomización en los conceptos, el carácter hegemónico del dME, la concepción de que la matemática es un conocimiento acabado y continuo, y la falta de marcos de referencia para la resignificación de la matemática escolar (Soto y Cantoral, 2014).

Se hace necesario un rediseño del dME para promover la construcción social del conocimiento matemático; la propuesta de la Socioepistemología es apoyar dicho rediseño en sus principios fundamentales, los cuales, sin formar una secuencia lineal, son: normativa de la práctica social, racionalidad contextualizada, relativismo epistemológico y significación progresiva.

En la tabla 1 se muestra la relación entre las características del dME, los principios de la Teoría Socioepistemológica y una propuesta de rediseño del dME. En esta tabla los elementos de la tercera columna tienen las características de principios de diseño de tareas en un marco de nivel intermedio, pues como mencionan Kieran, Doorman y Ohtani (2015), son los principios, heurísticas o herramientas teóricas explícitas que se utilizan para justificar un diseño de tarea o secuencia de tareas. Además estos prin- 
cipios de diseño se pueden aplicar a gran variedad de objetos, conceptos y procesos matemáticos, atendiendo a su construcción social -la tesis socioepistemológica acerca del conocimiento matemático-.

Tabla 1. Caracterización teórica del dME y la fundamentación para su rediseño.

\begin{tabular}{|c|c|c|}
\hline dME actual (Soto, 2010) & $\begin{array}{c}\text { Principios de la } \\
\text { Socioepistemología }\end{array}$ & Propuesta de dME \\
\hline $\begin{array}{c}\text { Carácter utilitario } \\
\text { La organización de la matemática } \\
\text { escolar ha antepuesto la utilidad } \\
\text { del conocimiento a cualquiera } \\
\text { de sus restantes cualidades. }\end{array}$ & $\begin{array}{l}\text { Normativa de la } \\
\text { práctica social } \\
\text { La significación de las } \\
\text { matemáticas } \\
\text { mediante el uso: } \\
\text { anidación de prácticas. }\end{array}$ & $\begin{array}{l}\text { Carácter funcional } \\
\text { El saber matemático escolar se organiza con base en el uso } \\
\text { del conocimiento y el funcionamiento cognitivo, didáctico, } \\
\text { epistemológico y social en la vida de los seres humanos, } \\
\text { reconociendo a las prácticas sociales en la base de la creación } \\
\text { del conocimiento: contexto de significación. }\end{array}$ \\
\hline $\begin{array}{l}\text { Atomización en los conceptos } \\
\text { No considera los aspectos sociales, } \\
\text { contextuales y culturales que permiten } \\
\text { la constitución del conocimiento. }\end{array}$ & $\begin{array}{l}\text { Racionalidad } \\
\text { contextualizada } \\
\text { La relación con el } \\
\text { saber es una } \\
\text { función contextual. }\end{array}$ & $\begin{array}{l}\text { Racionalidades contextuales diversas } \\
\text { Se reconocen, privilegian y potencian diversos tipos de } \\
\text { racionalidad relativos a la realidad en la que el individuo se } \\
\text { encuentre en un momento y lugar; desde el cual se construirá } \\
\text { conocimiento: aula extendida (contexto situado). }\end{array}$ \\
\hline $\begin{array}{l}\text { Carácter hegemónico } \\
\text { Supremacía de argumentaciones } \\
\text { y significados frente a otros. } \\
\text { Conocimiento acabado y continuo } \\
\text { La enseñanza de la matemática se } \\
\text { reduce a la mecanización de procesos } \\
\text { o memorización de los conceptos. }\end{array}$ & $\begin{array}{l}\text { Relativismo } \\
\text { epistemológico } \\
\text { La validez del saber } \\
\text { es relativa al } \\
\text { individuo y al } \\
\text { grupo cultural. }\end{array}$ & $\begin{array}{l}\text { Validación de saberes (conocimientos construidos) } \\
\text { El saber matemático escolar tiene diversas maneras de verse, } \\
\text { trabajarse, construirse y desarrollarse, concibiendo que la } \\
\text { validez del saber es relativa al individuo y al grupo cultural } \\
\text { en el cual este ha emergido y respecto a la racionalidad } \\
\text { contextualizada que este posea. }\end{array}$ \\
\hline $\begin{array}{l}\text { Falta de marcos de referencia } \\
\text { para su significación } \\
\text { Se ha soslayado el hecho de que la } \\
\text { matemática responde a otras prácticas } \\
\text { de referencia, donde se encuentran las } \\
\text { bases de significados naturales. }\end{array}$ & $\begin{array}{c}\text { Significación } \\
\text { progresiva } \\
\text { La significación } \\
\text { no es estática, es } \\
\text { funcional, relativa } \\
\text { y contextual. }\end{array}$ & $\begin{array}{c}\text { Pluralidad de prácticas de referencia } \\
\text { para la resignificación } \\
\text { La pluralidad de prácticas de referencia, su interacción } \\
\text { con diversos contextos y la propia evolución de la vida } \\
\text { del individuo o grupo resignificarán los saberes } \\
\text { hasta el momento construidos, enriqueciéndose } \\
\text { con nuevos significados. }\end{array}$ \\
\hline
\end{tabular}

Fuente: Reyes-Gasperini (2016), p. 44.

Para determinar los elementos de la construcción social del conocimiento matemático la Socioepistemología utiliza una herramienta teórica-metodológica denominada problematización del saber matemático, que se emplea para estudiar de forma articulada las distintas dimensiones de un saber matemático específico: dimensión epistemológica - circunstancias que hicieron posible la constitución del saber-, dimensión cognitiva 
-formas de apropiación y significación progresiva del conocimiento-, dimensión didáctica -cómo vive el saber en el sistema didáctico-y dimensión sociocultural -el uso situado del saber-.

A partir de la problematiación del saber se construyen herramientas que permitan analizar la interacción de los sujetos -individuales o colectivos- con el conocimiento matemático puesto en uso, a través de una situación de aprendizaje, que es una tarea o conjunto de tareas que propician la construcción del conocimiento matemático, poniendo en juego el contexto de significación (Reyes-Gasperini, 2016); en este sentido, una problematización del saber matemático para una pieza de conocimiento específica provee de un marco de dominio específico para la creación de situaciones de aprendizaje. De esta forma, los principios de diseño son los elementos que justifican de forma teórica la creación de la situación de aprendizaje como Instrumentos de intervención.

\section{Principios de diseño de tareas en MARCOS DE DOMINIO ESPECÍFICO SOCIOEPISTEMOLÓGICOS}

A continuación mostramos dos ejemplos de investigaciones que, desde la socioepistemología, a partir de una problematización del saber, proponen pautas para la elaboración de diseños de intervención; en particular los principios de diseño expuestos pertenecen a marcos de dominio específico, pues responden al estudio del estado estacionario en dos contextos distintos. El primer ejemplo se refiere a la serie trigonométrica de Fourier en su contexto de origen -la propagación del calor-, el segundo ejemplo trata acerca de la relación entre la propagación del calor y un contexto relativo al estado estacionario propio de la práctica de referencia de la ingeniería eléctrica.

\section{Ejemplo 1: La serie trigonométrica de Fourier en su contexto de origen}

Con la intención de significar las nociones matemáticas relacionadas con la serie trigonométrica de Fourier (STF), se realiza una problematización del saber matemático a través del estudio integrado de sus cuatro dimensiones, los detalles se encuentran en Romero (2016) y Farfán y Romero (2017).

A partir de dicha problematización se explica el funcionamiento del dME actual alrededor de la STF -ver tabla 2-, a través de la identificación de sus características principales (Soto y Cantoral, 2014). 
Tabla 2. Propuesta de rediseño del dME alrededor de la STF.

\begin{tabular}{|c|c|c|}
\hline dME actual alrededor de la STF & $\begin{array}{c}\text { Principios de la } \\
\text { Socioepistemología }\end{array}$ & Propuesta de dME para la STF \\
\hline $\begin{array}{c}\text { Carácter utilitario } \\
\text { La STF se presenta como un algoritmo } \\
\text { mecánico que permite resolver ciertos } \\
\text { problemas, lo que no permite su construcción } \\
\text { a partir de las características del contexto } \\
\text { histórico-social de su surgimiento. }\end{array}$ & $\begin{array}{c}\text { Normativa de la } \\
\text { práctica social } \\
\text { Esquema de anidación } \\
\text { de prácticas preliminar } \\
\text { para la STF } \\
\text { (Farfán y } \\
\text { Romero, 2017). }\end{array}$ & $\begin{array}{l}\text { Carácter funcional } \\
\text { La STF se presenta como una herramienta de } \\
\text { predicción al modelar e interpretar ciertos fenómenos } \\
\text { cercanos al sujeto (individual o colectivo), } \\
\text { reconociendo a la Prediciere como práctica social } \\
\text { que que norma la construcción de la STF, } \\
\text { mediante su uso culturalmente situado. }\end{array}$ \\
\hline $\begin{array}{l}\text { Atomización en los conceptos } \\
\text { Carencia de argumentaciones y significados } \\
\text { que provengan de la actividad humana, } \\
\text { pues no se toma en cuenta la práctica de } \\
\text { referencia que hace emerger a la STF } \\
\text { ni tampoco el contexto de quien aprende } \\
\text { (aula extendida). }\end{array}$ & $\begin{array}{c}\text { Racionalidad } \\
\text { contextualizada }\end{array}$ & $\begin{array}{c}\text { Racionalidades contextuales diversas } \\
\text { Considerar la evolución de lo trigonométrico; } \\
\text { esto permite la emergencia de argumentaciones } \\
\text { en el contexto de quien aprende (contexto situado). } \\
\text { Se debe resignificar a la función trigonométrica } \\
\text { para que surja la serie trigonométrica a partir } \\
\text { del estudio de la convergencia. }\end{array}$ \\
\hline $\begin{array}{c}\text { Carácter hegemónico } \\
\text { La STF sirve para aproximar una función por } \\
\text { serie trigonométrica (significado impuesto), } \\
\text { a través de un algoritmo mecánico para el } \\
\text { cálculo de sus coeficientes (predominio } \\
\text { del marco algebraico-analítico). } \\
\text { Conocimiento acabado y continuo } \\
\text { La STF es un algoritmo preestablecido } \\
\text { con carácter utilitario. Esto provoca } \\
\text { que deba ser memorizado y } \\
\text { aplicado a problemáticas específicas. }\end{array}$ & $\begin{array}{c}\text { Relativismo } \\
\text { epistemológico }\end{array}$ & $\begin{array}{c}\text { Validación de saberes } \\
\text { (conocimientos construidos) } \\
\text { Detrás de la STF existe diversidad de argumentaciones: } \\
\text { físicas, geométricas, analíticas y algebraicas. Por lo que } \\
\text { se deben considerar esta diversidad a la hora de } \\
\text { construir el conocimiento, ya que la validez del saber } \\
\text { es relativa al individuo y al grupo cultural en el cual } \\
\text { este ha emergido y respecto a la racionalidad } \\
\text { contextualizada que este posea. }\end{array}$ \\
\hline $\begin{array}{c}\text { Falta de marcos de referencia } \\
\text { para su significación } \\
\text { Existe la necesidad de identificar marcos } \\
\text { de referencia a partir de los cuales se } \\
\text { construyan bases de significados para las STF. }\end{array}$ & $\begin{array}{l}\text { Significación } \\
\text { progresiva }\end{array}$ & $\begin{array}{c}\text { Pluralidad de prácticas de referencia } \\
\text { para la resignificación } \\
\text { La construcción de la STF requiere del modelaje } \\
\text { e interpretación de un fenómeno estacionario } \\
\text { de variación periódica y acotada. }\end{array}$ \\
\hline
\end{tabular}

Fuente: Síntesis de la tabla presentada en Romero (2016), p. 87.

La tabla 2 es una adaptación de la tabla 1, directamente relacionada con una propuesta de rediseño para el dME alrededor de la STF, que da cuenta de su construcción social. Los elementos de la tercera columna de la tabla 2 se pueden considerar como principios de diseño en un marco de dominio específico, ya que estos son producto de un estudio relativo a la STF en su contexto de origen directamente. Además la problematización del saber realizada permitió identificar otro principio de diseño, que refiere a: 
Dos momentos importantes de construcción social de la STF: 1) el estudio de fenómenos estacionarios y 2) la representación de una función arbitraria en serie trigonométrica, esto en contra de la enseñanza usual. Puesto que, se suele privilegiar el segundo momento sin atender al primero, lo que provoca carencia de significados a partir de los contextos de uso. [Farfán y Romero, 2017, p. 501. El resaltado es nuestro]

Dado este principio de diseño, Farfán y Romero (2019) plantean una propuesta de situación de aprendizaje que consta de seis tareas, las primeras cinco corresponden al momento de estudio de fenómenos estacionarios para la construcción social de la STF y la sexta tarea corresponde a la representación de una función en serie trigonométrica. En dicho diseño se pone en funcionamiento un contexto físicogeométrico relativo a la astronomía, el modelo de movimiento planetario de los astrónomos alejandrinos -323 a.C.-30 a.C.-, para significar las nociones relacionadas con la STF. ${ }^{1}$ Esto aborda la necesidad de identificar distintas prácticas de referencia para la resignificación -pluralidad de prácticas de referencia para la resignificación (tabla 2)-.

Con el fin de ilustrar el funcionamiento de los principios de diseño, detallaremos las preguntas de la tarea \#3 de la situación de aprendizaje con su intencionalidad. Esta tarea tiene por objetivo significar la convergencia de series trigonométricas mediante la estabilidad de la trayectoria del planeta, caracterizándola mediante el límite de la sucesión de sumas parciales. Se espera que, al igual que en el trabajo de Fourier, el carácter estable del fenómeno provoque la necesidad de hablar de la convergencia de series trigonométricas -carácter funcional (tabla 2)-. En la figura 1 se observa la situación planteada.

Tarea \#3. Un modelo más general.

Llamemos a la Tierra $T$ y consideremos un cierto planeta $P$ cuya órbita corresponde a una superposición de movimientos circulares en torno de $T$, el modelo se comporta de la manera siguiente:

* E1 radio de la circunferencia número $n$ está dado por $\frac{\sqrt{n^{2} \pi^{2}+2-2(-1)^{2}}}{n^{2} \pi}$.

* La velocidad angular, en radianes por mes, de los puntos sobre las circunferencias primera, segunda, tercera, . . . son respectivamente $1,2,3, \ldots$

* Cuando el tiempo es $t=0$, el ángulo en posición estándar del punto con respecto al centro de la circunferencia número $n$ es $\theta_{n}=\arctan \left(\frac{1-(-1)^{n}}{n \pi}\right)$.

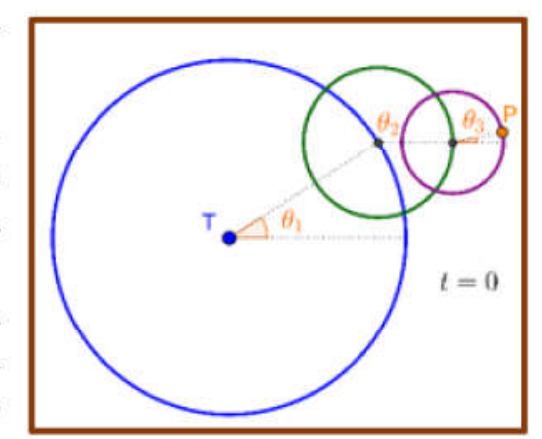

Figura 1. Situación planteada en la tarea \#3 de la situación de aprendizaje. Fuente: Elaboración propia a partir de lo presentado en Farfán y Romero (2019).

1 Para ampliar detalles acerca de este modelo del movimiento planetario se puede revisar Calles, Yépez y Peralta (2003). 
Esta tarea está dividida en dos partes, la primera busca que el estudiante comprenda cómo se comporta el sistema, y la relación que guarda con los datos suministrados por la situación planteada. Para empezar, en el inciso $a$-figura 2 - se pretende reconocer el comportamiento del movimiento de los puntos sobre cada circunferencia - racionalidad contextual diversa (tabla 2)-.

Parte I. Comprendiendo el modelo.

Con base en la situación planteada, realice lo que se le solicita a continuación:

a) Considere el modelo con dos circunferencias, como en la imagen adjunta. Determine la medida (en radianes) de los ángulos $\angle O T R$ y $\angle Q R P$, después de $t$ meses.

b) Regresando al modelo utilizando dos circunferencias y agregando un sistema de coordenadas, en el cual $T$ esté en el punto $\left(\frac{\pi}{4}, \frac{\pi}{4}\right)$.

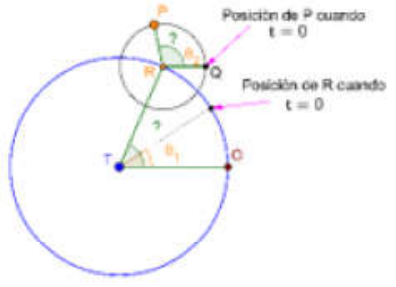

Determine las coordenadas $\left(x_{1}, y_{1}\right)$ de $R$ y las coordenadas $\left(x_{2}, y_{2}\right)$ de $P$, para cualquier valor de $t$. Determine además la distancia de $P$ a $T$, para cualquier $t$.

c) Considere ahora el modelo utilizando tres circunferencias. Determine una fórmula que permita calcular las coordenadas $\left(x_{3}, y_{3}\right)$ del planeta $P$, para cualquier valor de tiempo $t$.

Figura 2. Tarea \#3 - parte I de la situación de aprendizaje. Fuente: Elaboración propia a partir de lo presentado en Farfán y Romero (2019).

En las preguntas $b$ y $c$-figura 2 - se espera que los estudiantes identifiquen las razones trigonométricas dibujando triángulos rectángulos sobre las figuras proporcionadas; sin embargo, es necesario que la función trigonométrica esté construida previamente para generalizar a cualquier valor del tiempo (Montiel, 2011). De esta manera se pretende construir las primeras tres sumas parciales de dos series trigonométricas, pero con un significado asociado al fenómeno, las coordenadas del planeta - racionalidad contextual diversa y carácter funcional (tabla 2)-.

La segunda parte de la tarea busca, mediante la manipulación del número de circunferencias, visualizar la estabilidad del sistema. Para esto se proporciona un applet de GeoGebra donde se puede analizar cómo cambia la trayectoria del planeta al aumentar el número de circunferencias -figura 3-. 


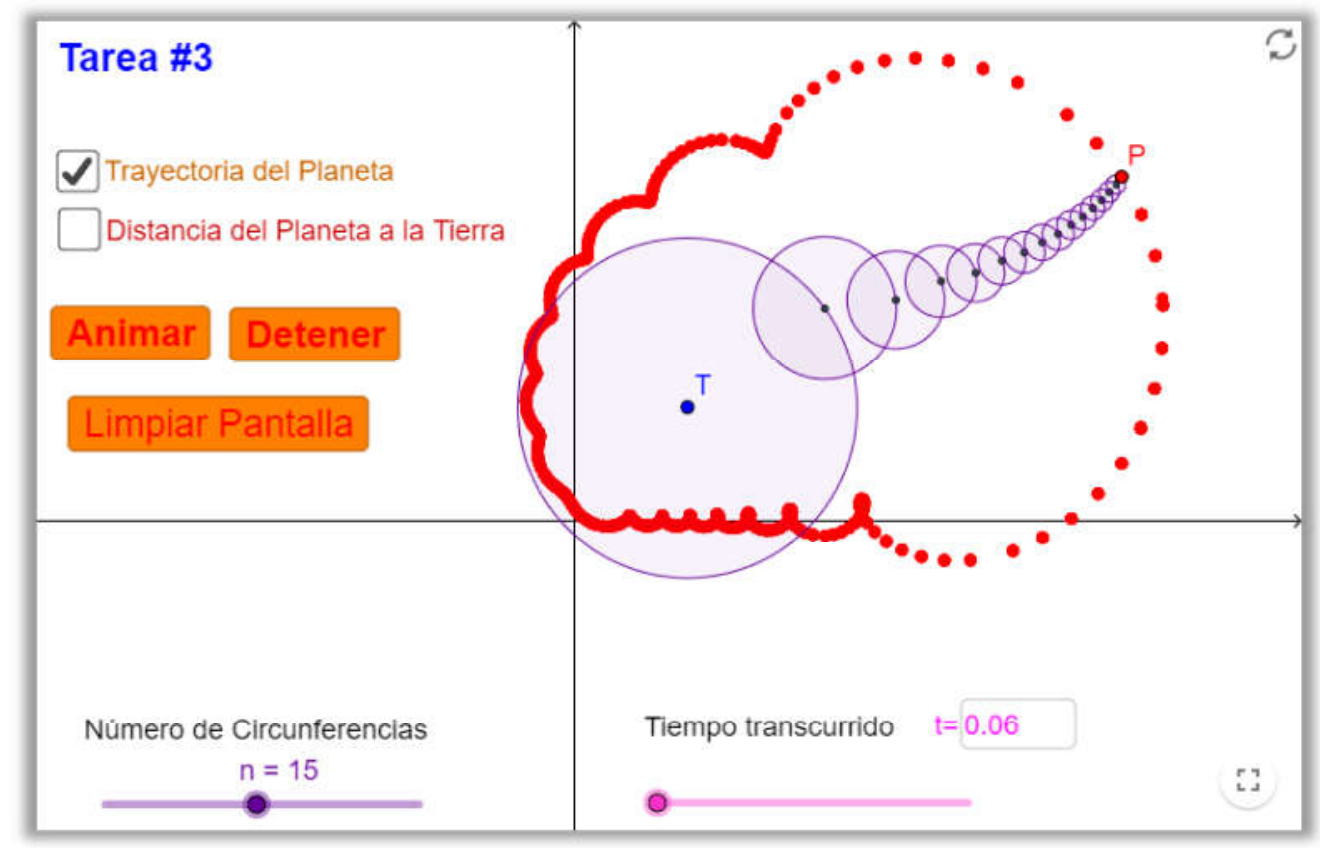

Figura 3. Applet de la tarea 3 - parte II (puede consultarse en https://ggbm.at/tJwCF8um). Fuente: Elaboración propia a partir de lo presentado en Farfán y Romero (2019).

Para las preguntas planteadas en la figura 4, en términos generales, se busca primero que el estudiante identifique que la trayectoria del planeta se estabiliza conforme se agregan más circunferencias, mediante argumentos como "cuando hay muchas circunferencias casi no cambia la forma de la trayectoria conforme se agregan más", "tiende a parecerse a una..." -validación de saberes (tabla 2)-. Luego, con un análisis numérico de la variación de una suma parcial a otra, se busca que el estudiante se percate de que la trayectoria del planeta no es estable para todos los valores del tiempo -validación de saberes (tabla 2)-. Seguidamente se pasa a matematizar el fenómeno y la interpretación de la matemática involucrada en el fenómeno físico, relacionar la estabilidad de la trayectoria del planeta con el límite de las sumas parciales involucradas en la fórmula calculada -validación de saberes y racionalidad contextual diversa (tabla 2)-. 
Parte II. ¿Y si aumentamos el número de epiciclos?

A partir de lo observado en el applet proporcionado responde:

a) ¿Cómo cambia la trayectoria del planeta conforme se agregan más y más circunferencias? Explica con tus propias palabras.

b) ¿Cómo cambia la distancia del planeta $P$ a la Tierra conforme se agregan más y más circunferencias para cada instante de tiempo? Explica con tus propias palabras. c) Considere ahora el modelo utilizando $n$ circunferencias. Determine una fórmula que le permita calcular las coordenadas $\left(x_{n}, y_{n}\right)$ del planeta $P$ en el sistema coordenado, en cualquier tiempo $t$.

d) A partir de sus respuestas a las preguntas (a) y (b) ¿cómo es el comportamiento de la fórmula obtenida en la pregunta (c)? Explique su respuesta.

Figura 4. Tarea \#3 - parte II, incisos del $a$ al $d$ de la situación de aprendizaje.

Fuente: Elaboración propia a partir de lo presentado en Farfán y Romero (2019).

Las siguientes preguntas de esta parte de la tarea se basan en el análisis de otro applet de GeoGebra -figura 5- en el que se muestra, por un lado, el modelo del movimiento de un planeta $P$ alrededor de la Tierra $T$ y, por otro lado, el cambio de las coordenadas del planeta $P$ conforme transcurre el tiempo.

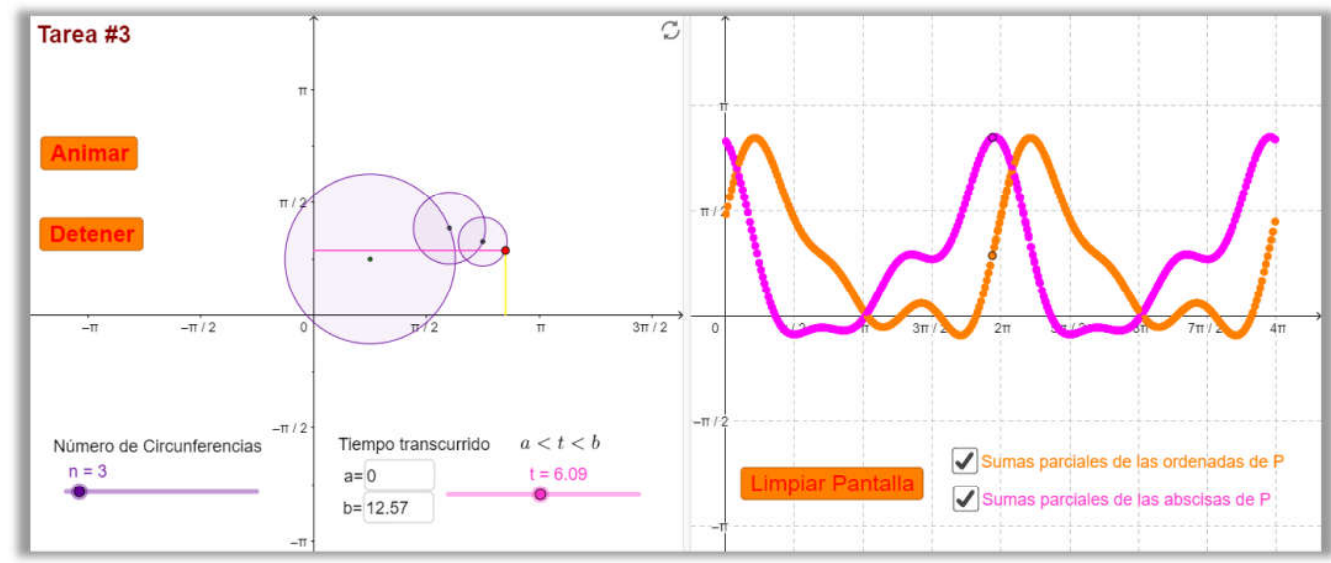

Figura 5. Segundo applet de la tarea 3 - parte II (puede consultarse en https://ggbm.at/Kwx2Mpcc).

Fuente: Elaboración propia a partir de lo presentado en Farfán y Romero (2019).

En general, se espera con estas preguntas - figura 6- la interpretación de la matemática involucrada en el fenómeno físico, relacionar la estabilidad de la trayectoria del planeta con la convergencia de las series involucradas en la fórmula calculada para la distancia de planeta $P$ a la Tierra -carácter funcional y validación de saberes (tabla 2)-. 
Parte II. ¿Y si aumentamos el número de epiciclos?

A partir de lo observado en el applet proporcionado responde:

e) ¿Cómo es el comportamiento de las sumas parciales de las abscisas? ¿Y el de las ordenadas? Explica con tus propias palabras. ¿Qué relación existe entre estas respuestas y las de las preguntas (a) y (b)?

f) Note que las fórmulas obtenidas corresponden a sumas parciales de series trigonométricas. Con base en el mismo applet y tu respuesta a la pregunta anterior ¿podrías asegurar si la serie de las ordenadas converge o diverge? En caso de que converja ¿podrías identificar su valor de convergencia? Explica tus respuestas.

g) Si se cambia el rango de valores de $t$, para todos aquellos en los que $t \geq 0$. ¿Cuál es el valor de convergencia de la serie de las ordenadas del planeta $P$ ?

h) ¿Tiene sentido en nuestro modelo considerar valores de $t$ negativos? ¿Cuál sería el valor de convergencia de la serie de las ordenadas del Planeta $P$ en caso de considerar a $t \in \mathbb{R}$ ?

i) Considere ahora la serie de las ordenadas de $P$. Utilizando identidades trigonométricas, escríbala en la forma:

$$
\frac{a_{0}}{2}+\sum_{k=1}^{n} a_{k} \cos (k t)+b_{k} \operatorname{sen}(k t)
$$

Es decir, identifique los valores de $a_{0}, a_{k}$ y $b_{k}$.

Figura 6. Tarea \#3 - parte II, incisos del $e$ al $i$ de la situación de aprendizaje.

Fuente: Elaboración propia a partir de lo presentado en Farfán y Romero (2019).

Luego, la serie, como objeto matemático, posee ciertas características que no tienen sentido en el fenómeno que se está modelando. En este caso dicha característica es la condición de periodicidad, la cual se da en toda la recta real, pero en el fenómeno no tiene sentido hablar de valores negativos de tiempo - racionalidad contextual diversa (tabla 2)-, por lo que se trata de provocar que la periodicidad sea un resultado del trabajo con la serie y no una condición de partida.

Se muestra entonces cómo los principios de diseño construidos a partir de la problematización del saber permiten guiar la organización de la situación de aprendizaje. Sumado a lo anterior, y con el propósito de ahondar en otras prácticas de referencia necesarias para la resignificación (tabla 2), el siguiente ejemplo hace énfasis en la noción de estado estacionario en la práctica de la ingeniería eléctrica.

\section{Ejemplo 2: La noción del estado estacionario en ingeniería eléctrica}

En este ejemplo se toma una investigación que se realiza dentro del marco de la Socioepistemología, que tiene el objetivo de caracterizar las nociones acerca del estado estacionario de estudiantes de ingeniería eléctrica con fenómenos que se encuentran en dicho estado. Esta investigación utiliza como punto de partida los estudios realizados por Farfán (2012), en los que la autora identificó que el contexto situacional donde emerge la STF fue la propagación del calor -en la Teoría Analítica del Calor de 
Fourier-, y para caracterizar las nociones acerca del estado estacionario se realizó una problematización del saber en la que se buscó la relación entre la propagación de calor y los fenómenos eléctricos de donde se determinan los principios de diseño que posteriormente son utilizados para configurar un instrumento de intervención didáctica.

\section{La problematización del saber: la analogía entre el calory la electricidad}

Según Narasimhan (1999), la Teoría Analítica del Calor de Fourier fue una obra que impulsó el desarrollo científico en diversas ramas de la ciencia durante el siglo XIX. Las ciencias eléctricas en particular utilizaron ampliamente el trabajo de Fourier para establecer modelos que dieran explicación al equilibrio electrostático y las condiciones para la conducción de la electricidad. La problematización consistió en dos fases: 1) una revisión de planes y programas de estudio de ingeniería eléctrica donde se identificaron las asignaturas donde aparece la STF y 2) un análisis histórico-epistemológico de donde se obtuvieron los principios de diseño para el instrumento de intervención didáctica.

En la fase 1 de la problematización identificamos que en los planes de estudio de ingeniería electrónica, mecatrónica y electromecánica de una universidad del norte de México, la STF aparece como un contenido matemático a abordar en los programas de asignaturas como Señales y sistemas, Circuitos eléctricos y Sistemas electrónicos de potencia -segundo, tercero y cuarto año en los planes de estudio respectivamente-; en dichas asignaturas el contenido es visto como una herramienta/algoritmo matemático que permite, entre otras cosas, representar señales eléctricas en el tiempo a través de una sumatoria de funciones senoidales y analizar el estado estacionario de circuitos eléctricos (Hinojos y Farfán, 2017a), lo cual es consistente con lo reportado en la tabla 2, columna 1, referente al carácter hegemónico y al conocimiento continuo y acabado del dME alrededor de la STF.

Dado que tanto en circuitos eléctricos como en la propagación del calor se estudia el estado estacionario, y es de este contexto situacional de donde emerge la STF como un objeto matemático que es utilizado en asignaturas de ingeniería eléctrica, reconocemos a la ingeniería como una práctica de referencia -pues tenemos un uso del conocimiento matemático, un usuario y un contexto- para la resignificación del estado estacionario y planteamos como pregunta de investigación: ¿Qué relación existe entre el calor y la electricidad?

La pregunta de investigación planteada se retoma en la fase 2, en la cual se realizó una búsqueda en obras originales de científicos del siglo XIX referentes a las ciencias eléctricas, con el criterio de que dichas obras hicieran referencia al trabajo de Fourier. Con este criterio, se encontraron las obras de Ohm (1827), Thomson (1872) y Maxwell (1881) de las cuales se realizó un análisis histórico-epistemológico, obteniendo lo siguiente: 
- Die galvanische Kette, mathematisch bearbeitet, de G. S. Ohm (1827). En este libro, el autor realiza la matematización de la conducción eléctrica-galvanismo-a través de la utilización de una analogía con el trabajo de Fourier, la experimentación en condiciones de laboratorio y el análisis de circuitos eléctricos - de algunos centímetros de longitud- alimentados con pilas voltaicas con ciertas idealizaciones del fenómeno. $\mathrm{Al}$ realizar el análisis de circuitos eléctricos, Ohm consideró que los alambres que lo forman son cuerpos prismáticos y libres de impurezas, indicando que la sustancia eléctrica-fluido galvánico- no escapa del interior de este, mientras se alcanza un estado de equilibrio en el flujo.

La analogía utilizada por Ohm se caracteriza por dos consideraciones: 1) el fenómeno de conducción de electricidad se encuentra en estado estacionario -el estado de equilibrio en el flujo- y 2) la ecuación diferencial parcial obtenida al final de su análisis es igual a la mostrada por Fourier en la teoría analítica del calor; esto nos permitió identificar que los modelos matemáticos son análogos dado que ambos fenómenos -la conducción eléctrica y la propagación de calor- se encuentran en estado estacionario.

- On the motion of heat in homogeneous solid bodies, and its connexion with the mathematical theory of electricity y On the mathematical theory of electricity in equilibrium, de W. Thomson (1872), son dos artículos publicados por Thomson donde hace referencia al trabajo de Fourier para explicar teóricamente el estado de equilibrio electrostático. En el primero -On the motion of heat- el autor menciona las características físicas y condiciones que hacen análogos a ambos fenómenos, indicando las correspondencias entre ambos cuando están en estado estacionario -tanto física como matemáticamente-; en el segundo -On the mathematical theory - retoma lo desarrollado en el primer trabajo a través de un ejemplo de un sistema de cargas eléctricas en equilibrio electrostático y un sistema térmico en estado estacionario, concluyendo que el estado estacionario de las temperaturas -el equilibrio térmico en este caso- y el equilibrio electrostático son matemáticamente iguales. Interpretamos de los argumentos de Thomson que consideró al equilibrio térmico y al equilibrio electrostático como fenómenos de estado estacionario análogos dadas sus características físicas y que esto permite que puedan utilizarse los mismos fundamentos matemáticos para modelarlos.

- A treatise on electricity and magnetism, de J. C. Maxwell (1881). El primer volumen de este tratado - escrito con la intención de utilizarse como libro de texto- describe las características de fenómenos electromagnéticos y la conducción de electricidad a través de alambres telegráficos - circuitos eléctricos de varios kilómetros de longitud-; es en el análisis de la transmisión de mensajes telegráficos donde el autor utiliza una analogía con la teoría analítica del calor para modelar matemáticamente el problema, identificando que, a diferencia del calor, la electricidad no es una manifestación de la energía: bajo ciertas condiciones, el flujo de electricidad corresponde al flujo de calor, el potencial eléctrico corresponde a la temperatura, y la electricidad fluye del potencial mayor 
al menor, como ocurre con el calor, que fluye de la temperatura mayor hacia la menor. Maxwell además realiza el análisis de un circuito eléctrico equivalente al alambre telegráfico y las condiciones de estado estacionario que surgen al enviar un mensaje a través de este, obteniendo una ecuación diferencial parcial con la misma estructura que la ecuación de Fourier (detalles en Hinojos y Farfán, 2017b).

Como se describió anteriormente, los tres científicos mencionados utilizaron analogías entre el calor y la electricidad cuando ambos tipos de fenómenos se encuentran en estado estacionario, enmarcados en la racionalidad física y paradigmas de su época, utilizando la misma expresión matemática de Fourier (detalles en Hinojos y Farfán, 2017b); además se identificó la presencia de un obstáculo epistemológico -en el sentido de Bachelard- denominado "obstáculo sustancialista", el cual se describe como un paradigma del pensamiento científico del siglo XIX que se caracteriza por anteponer la experiencia sensorial-física a los razonamientos inductivos y deductivos o a la evidencia empírica obtenida de la experimentación con fenómenos físicos (Hinojos y Farfán, 2018).

Este análisis histórico-epistemológico permitió establecer la hipótesis epistemológica de la investigación: El conocimiento matemático relativo al estado estacionario se construye transitando entre los paradigmas estático y dinámico con el establecimiento de analogías materialsemiformal-formal del calor y la electricidad. Esto es válido desde el punto de vista teórico dado el principio de la racionalidad contextualizada, mismo que permite configurar nuestros principios de diseño:

- Trabajar con las analogías entre el calor y la electricidad de las obras de científicos del siglo XIX.

- Utilizar al obstáculo sustancialista como un elemento de confrontación de las nociones de electricidad.

- Organizar el diseño iniciando con las analogías físicas entre los fenómenos y posteriormente con la matematización de estas.

\section{El diseño de intervención}

Tomando como base lo anterior, consideramos que, aunque cronológicamente $\mathrm{Ohm}$ (1827) estableció la analogía entre calor y electricidad y su matematización antes que Thomson (1872) y Maxwell (1881), este científico realizó su trabajo inmerso en un paradigma dinámico - el galvanismo- en el que la analogía y matematización son más complejas, mientras que la analogía de Thomson (1872) fue realizada en un paradigma estático -la electrostática-.

La organización del diseño comienza estableciendo una analogía material -las similitudes físicas entre los fenómenos- en paradigma estático que transita hacia una analogía formal -considerando la matematización de los fenómenos-en un paradigma dinámico. Esto es, se comienza con una analogía que establece las equivalencias entre las racionalidades físicas de los fenómenos en un paradigma estático -del equilibrio 
electrostático y el calor en estado estacionario- que evoluciona a lo largo de una serie de tareas hacia una analogía que, además de la física, también considera la matematización del estado estacionario de los fenómenos en un paradigma dinámico.

La confrontación con el obstáculo sustancialista se da a lo largo de las tareas a través de la formalización de las analogías en tres fases: 1) material, enfocada únicamente en las equivalencias físicas entre los fenómenos; 2) semiformal, en la que tanto las similitudes físicas como el trabajo matemático trabajan a la par, pero el problema no se resuelve sin ambas al mismo tiempo, y 3) formal, en la que se utilizan tanto las similitudes físicas como el trabajo matemático, pero el modelo matemático es autónomo del conocimiento de la física.

Con base en lo anterior, el diseño entonces queda fundamentado en los principios obtenidos de la problematización del saber que configuraron cuatro tareas, una por cada obra revisada -Thomson (1872), Ohm (1827) y Maxwell (1881) - y una más en un contexto específico de la población destino -el estudio de sistemas convertidores de energía-, todo esto dentro de la práctica de referencia de la ingeniería eléctrica.

A modo de ejemplo mostramos un extracto de la tarea 3 -la tarea está basada en la obra de Maxwell (1881) y se divide en cuatro partes-, donde se ilustra el trabajo con la analogía formal -figuras 7 a $10-$.

a) Considere el sistema de cuerpos de la figura, donde ambos son conductores de calor y electricidad, pero el hilo es aislante para ambos:

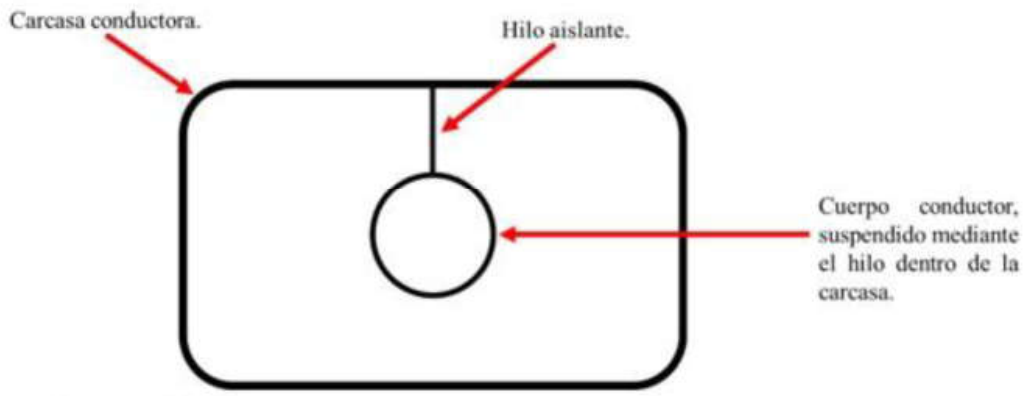

Supongamos los siguientes casos:

- Se somete a la carcasa conductora a un incremento de temperatura. ¿Cómo cambiará la temperatura del cuerpo que se encuentra en el interior de la carcasa?

- Se saca el cuerpo de la carcasa y se dejan ambos separados en contacto con el medio ambiente. ¿Cómo cambiará la temperatura de ambos conforme pasa el tiempo?

- En la misma configuración inicial, se somete a la carcasa conductora a un proceso de electrificación, de tal manera que su carga eléctrica se incremente y, en consecuencia, su potencial eléctrico también. ¿Qué ocurrirá con el cuerpo contenido dentro de la carcasa?

- Se saca el cuerpo de la carcasa y se dejan ambos separados en contacto con el medio ambiente. ¿Cómo cambiará la carga y el potencial de ambos conforme pasa el tiempo?

Figura 7. Analogía material de la tarea 3 -inciso a- de Maxwell.

Fuente: Elaboración propia con base en Hinojos y Farfán (2017b). 
En la figura 7 se muestra un problema donde se realiza la comparación entre dos fenómenos, uno de calentamiento/enfriamiento y otro de carga/descarga eléctrica; la intención de este problema es que al resolverlo el estudiante profundice en las similitudes y diferencias de la racionalidad física entre ambos, de tal forma que la analogía material -que se establece previamente- se retome para formalizarla.

Se toma una placa gruesa de cualquier material conductor y se le suministra calor a una de sus caras, provocando que exista un incremento de su temperatura y, que el calor fluya desde dicha cara hacia la contraria.

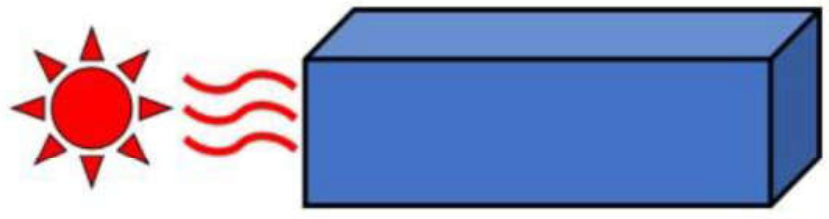

Súbitamente, la cara donde fue suministrado el calor se enfría a la misma temperatura que la cara del lado contrario y se deja a la placa actuando con el medio ambiente.

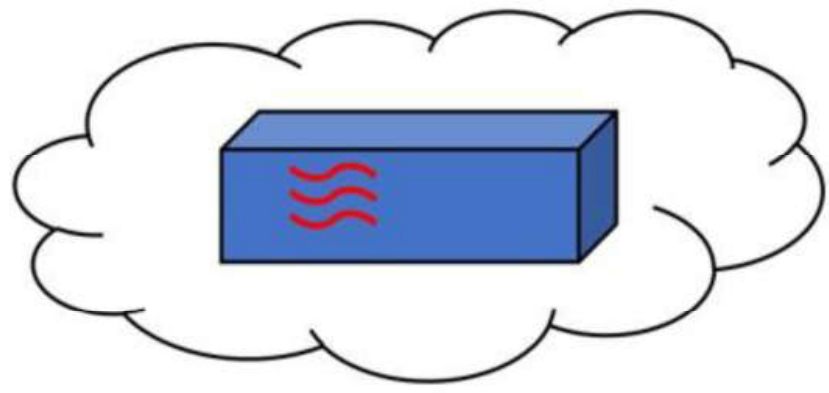

- ¿Cómo cambiará la temperatura de la placa conforme pasa el tiempo?

- ¿Cuál cara de la lámina tendrá la mayor temperatura en un tiempo corto? ¿por qué?

- ¿Cómo podría hacerse un experimento equivalente con un fenómeno eléctrico?

Figura 8. Comparación entre fenómenos de absorción de la tarea 3 -inciso b- de Maxwell. Fuente: Elaboración propia con base en Hinojos y Farfán (2017b), Maxwell (1881, p. 417-422).

Lo mostrado en la figura 8 se basa en un ejemplo expuesto por Maxwell (1881, p. 419); la intención de este inciso es que se establezca la equivalencia entre un fenómeno térmico que presenta absorción y mantenimiento de calor con algún fenómeno eléctrico; el fenómeno equivalente -basado igualmente en Maxwell (1881)- se da en el contexto de la transmisión de mensajes por telégrafo trasatlántico, como se muestra en la figura 9. 
c) Considere el siguiente experimento:

Nos encontramos en el año 1866, y se requiere enviar un mensaje urgente desde Londres hasta Nueva York. Las comunicaciones satelitales no existian en dicha época, por lo que la solución consiste en enviarlo por medio del telégrafo transatlántico.

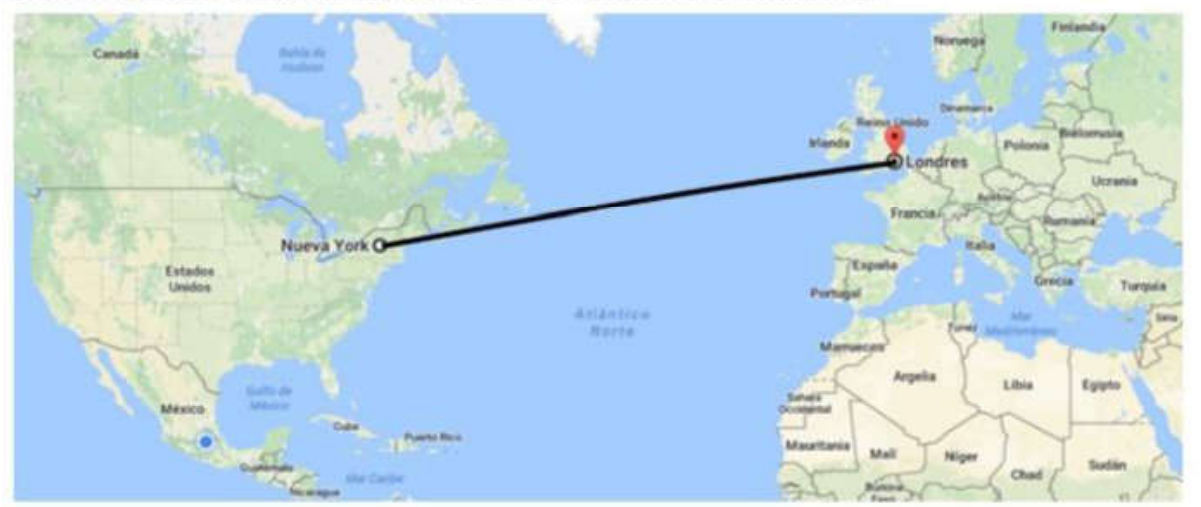

1. Describa con sus palabras o por medio de dibujos y diagramas, ¿cómo operaría el telégrafo para enviar dicho mensaje?

Figura 9. Indicios para la matematización del

fenómeno eléctrico de la tarea 3 -inciso c- de Maxwell.

Fuente: Elaboración propia con base en Hinojos y Farfán (2017b), Maxwell (1881, p. 419-422).

Se espera que para resolver lo planteado en la figura 9 los estudiantes utilicen su conocimiento previo de física y hagan alusión a que la electricidad se transmite a altas velocidades, sin embargo, dada la distancia que separa a ambos puntos en la comunicación y considerando que los telégrafos no realizan una comunicación instantánea, esto permite confrontar el obstáculo sustancialista al encontrar un contraejemplo de la intuición acerca del comportamiento de la electricidad instantáneo. En una sección subsecuente de la tarea se muestra el análisis del circuito eléctrico equivalente al telégrafo -ver figura 10- y mediante este análisis se obtiene la ecuación diferencial parcial que modela la transmisión de mensajes a través del telégrafo, separando las racionalidades física y matemática para formalizar la analogía entre los fenómenos. 


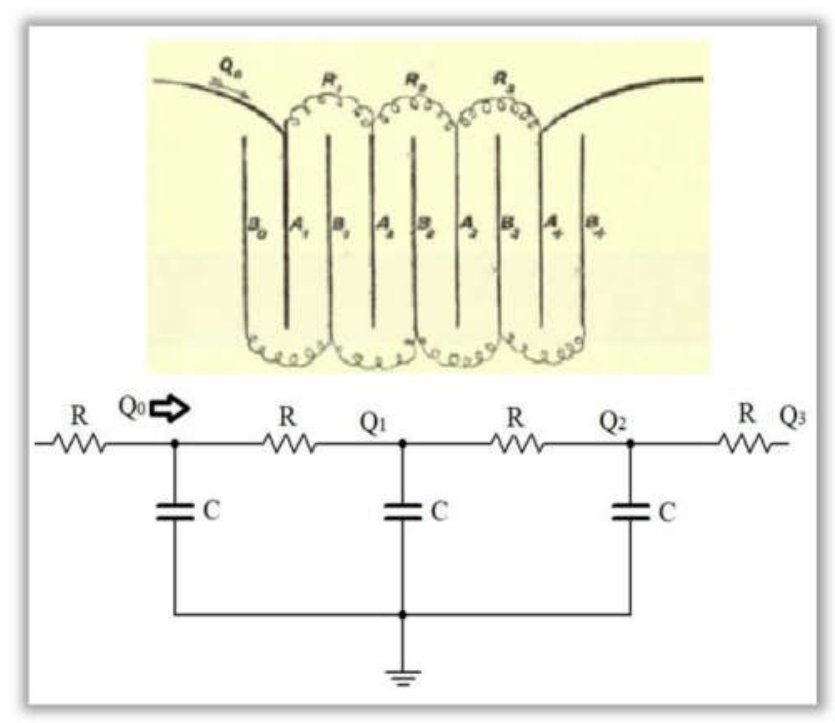

Figura 10. Reinterpretación del circuito equivalente al telégrafo. Fuente: Elaboración propia con base en Maxwell (1881, p. 420).

\section{Conclusiones}

Este escrito, como reflexión teórica, busca contribuir a la forma en cómo se presenta la fundamentación de los diseños de intervención didáctica en Socioepistemología, concretamente lo relacionado con las situaciones de aprendizaje. Consideramos que la caracterización y explicitación de los principios del diseño es necesaria para justificar cómo se configuran los diseños a partir de una problematización del saber.

Según Cantoral, Montiel y Reyes-Gasperini (2015), una propuesta de rediseño del dME debe poseer las siguientes características: carácter funcional, racionalidades contextuales diversas, validación de saberes y pluralidad de prácticas de referencia. En este sentido los ejemplos muestran cómo la problematización permite construir principios de diseño en marcos de dominio específico y que estos no solo dependen de los objetos/conceptos en juego, sino también de su uso culturalmente situado (Cantoral y Farfán, 2003).

En el sentido de Kieran, Doorman y Ohtani (2015), consideramos a los cuatro principios de la Teoría Socioepistemológica -racionalidad contextualizada, relativismo epistemológico, significación progresiva y normativa de la práctica social- dentro de los principios de diseño como un marco de nivel intermedio, dado que son los fundamentos filosóficos explícitos de la teoría que nos permiten realizar propuestas para el rediseño del dME; mientras que la problematización del saber constituye un marco de dominio específico, dado que se enfoca en las dimensiones de un saber matemático específico en el cual se abordan conceptos, procedimientos o procesos de la matemática particular que se estudia. 
Lo anterior se ilustra en los ejemplos expuestos: en el primero se construyen principios de diseño en un marco de dominio específico - con la problematización de la STF- que se ven directamente relacionados con los principios de diseño de nivel intermedio -lo mostrado en las tabla 1 y 2 -. En el segundo se muestra cómo desde el marco de referencia de la ingeniería eléctrica puede darse la resignificación de nociones de estado estacionario con el marco de dominio específico de la problematización de estas nociones en su contexto histórico situado.

Esto da evidencia de que en Socioepistemología la problematización del saber matemático juega un papel importante en la construcción de principios de diseño en marcos de dominio específico, pues es a partir de esta que se proponen rediseños del dME, plasmados principalmente en diseños de intervención.

\section{Agradecimientos}

Los autores queremos agradecer a la Dra. Gisela Montiel Espinosa del Cinvestav-IPN por su revisión y comentarios que permitieron pulir detalles del escrito.

\section{REFERENCIAS}

Calles, A., Yépez, E. y Peralta, J. (2003). El análisis de Fourier de las trayectorias planetarias y el modelo copernicano del sistema solar. Revista Mexicana de Física, 49(3), 283-289.

Cantoral, R. (2013). Teoría socioepistemológica de la matemática educativa. Barcelona: Gedisa.

Cantoral, R. y Farfán, R. (2003). Matemática educativa: una visión de su evolución. Revista Educación y Pedagogía, 15(35), 203-214.

Cantoral, R., Montiel, G. y Reyes-Gasperini, D. (2015). Análisis del discurso matemático escolar en los libros de texto, una mirada desde la teoría socioepistemológica. Avances de Investigación en Educación Matemática, (8), 9-28.

Chevallard, Y. (1997). La transposición didáctica: del saber sabio al saber enseñado. Buenos Aires: Aique.

Farfán, R. y Romero, F. (2017). Construcción social del conocimiento matemático: la serie trigonométrica de Fourier desde la socioepistemología. Perspectivas da Educação Matemática - INMA/UFMS, 10(23), 483-503.

Farfán, R. y Romero, F. (2019). Situación de aprendizaje para la serie trigonométrica de Fourier desde la teoría socioepistemología. Acta Scientiae, 21(2), 28-48. DOI: 10.17648/acta.scientiae.v21iss2id5019.
García, F. (2019). Introducción a 'Diseño de tareas en educación matemática: una diversidad de marcos teóricos'. Avances de Investigación en Educación Matemática, 15, 1-4.

Hinojos, J. y Farfán, R. (2017a). Breve recorrido por el discurso matemático escolar de la serie de Fourier en el contexto del ingeniero en electrónica. Acta Latinoamericana de Matemática Educativa, 31(1), 584-591.

Hinojos, J. y Farfán, R. (2017b). Acerca de las nociones de estabilidad en electricidad, la relación entre el calor y la electricidad. Revista de História da Educação Matemática, 3(3), 68-100.

Hinojos, J. y Farfán, R. (2018). La analogía entre el calor y la electricidad. Una base para confrontar el obstáculo epistemológico sustancialista en la electricidad en escuelas de ingeniería. Acta Latinoamericana de Matemática Educativa, 30, 838-846.

Kieran, C., Doorman, M. y Ohtani, M. (2015). Frameworks and principles for task design. En A. Watson y M. Ohtani (eds.). Task design in mathematics education: An ICMI study 22 (pp. 19-81). Nueva York: Springer.

Maxwell, J. (1881). A treatise on electricity and magnetism. Volume 1. Reino Unido: Cambridge University Press. 
Montiel, G. (2011). Construcción de conocimiento trigonométrico: un estudio socioepistemológico. México: Díaz de Santos.

Narasimhan, T. (1999). Fourier's heat conduction equation: History, influence, and connections. Reviews of Geophysics, 37(1), 151-172.

Ohm, G. (1827). Die galvanische Kette, mathematisch bearbeitet. Alemania.

Reyes-Gasperini, D. (2016). Empoderamiento docente y socioepistemología: un estudio sobre la transformación educativa en Matemáticas. Barcelona: Gedisa.

Romero, F. (2016). Construcción social de la serie trigonométrica de Fourier: pautas para un diseño de intervención en el aula [Tesis de maestría]. Centro de Investigación y de Estudios Avanzados del Instituto Politécnico Nacional, México. DOI: 10.13140/RG.2.2.14118.63048.
Soto, D. (2010). El discurso matemático escolar y la exclusión: una visión socioepistemológica [Tesis de maestría]. Centro de Investigación y de Estudios Avanzados del Instituto Politécnico Nacional, México.

Soto, D. y Cantoral, R. (2014). Discurso matemático escolar y exclusión. Una visión socioepistemológica. Bolema, 28(50), 1525-1544. DOI: 10.1590/1980-4415v28n50a25.

Thomson, W. (1872). Reprints of papers on electrostatics and magnetism. Reino Unido: Macmillan \& Co.

Whatson, A. y Ohtani, M. (eds.) (2015). Task design in mathematics education: An ICMI study 22. Nueva York: Springer.

Cómo citar este artículo:

Hinojos Ramos, J. E., Romero Fonseca, F. W., y Farfán Márquez, R. M. (2020). Principios de diseño de tareas en socioepistemología. IE Revista de Investigación Educativa de la REDIECH, 11, e708. doi: https://doi.org/10.33010/ ie_rie_rediech.v11i0.708. 